\title{
Cupric Oxide Nanoleaves for the Oxidative Degradation of Methyl Orange without Heating or Light
}

\author{
Cesar L. Londoño-Calderón, Sandra Menchaca-Nal, Nora J. François, * Laura G. Pampillo, \\ and Pablo Froimowicz*
}

Cite This: https://dx.doi.org/10.1021/acsanm.0c00283

Read Online

\begin{abstract}
ACCESS 1
Llll Metrics \& More

Article Recommendations

ABSTRACT: Low-cost 2D cupric oxide nanoleaves ( $\mathrm{CuO}$ NLs) are straightforwardly synthesized at room temperature by precipitation varying the addition method of the alkali. No further treatments are necessary to obtain high purity NLs. The effect of the different addition methods of alkali on the morphological, structural, vibrational, and optical properties is studied by field emission scanning electron microscopy (FESEM), X-ray diffraction (XRD), and Fourier transform infrared (FT-IR) and ultraviolet-visible (UV-vis) spectroscopies. NLs grown by alkali addition in a dropwise manner are on average 281, 178, and $17 \mathrm{~nm}$ long, wide, and thick, respectively, and composed of crystallites of $14 \mathrm{~nm}$ corresponding to the crystallographic planes (111)/

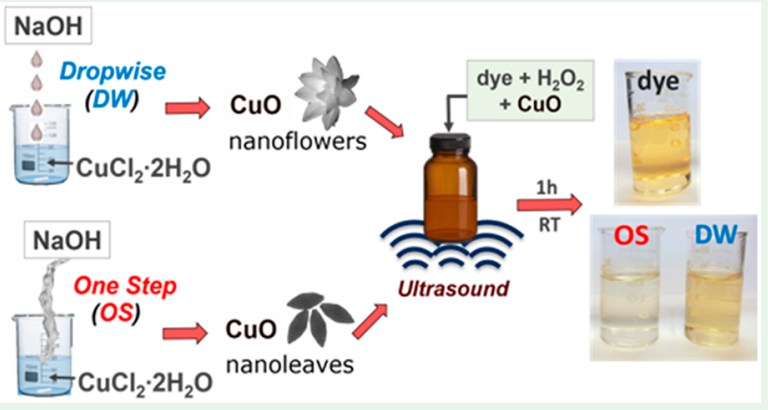
(002) and (111)/(200). NLs obtained by this method agglomerate forming flower-like nanostructures, exhibiting indirect band gap energy of $1.21 \mathrm{eV}$. NLs grown by alkali addition in a one-step manner are on average significantly bigger, being 602,219 , and $26 \mathrm{~nm}$ long, wide, and thick, respectively, composed of crystallites of 19 and $16 \mathrm{~nm}$ corresponding to the crystallographic planes $(\overline{1} 11) /(002)$ and $(111) /(200)$, respectively. These NLs agglomerate randomly with no predominant form observed, exhibiting indirect band gap energy of $1.39 \mathrm{eV}$. The addition method of alkali does not influence the average crystallite size of NLs, whereas the microstrain distribution is sensitive to the initial concentration of $\mathrm{OH}^{-}$ ions. Our results suggest that an indirect electronic transition between the valence and conduction bands might be more feasible than a direct one. NLs grown by the one-step method present the highest efficiency as catalyst toward catalytic oxidative degradation of the methyl orange dye with no heating and without the influence of light. Finally, this catalyst is easily recycled several times preserving its high catalytic activity.
\end{abstract}

KEYWORDS: chemical design, nanoparticles, nanoleaves, cupric oxide, catalytic degradation

\section{INTRODUCTION}

Nanostructured cupric oxide $(\mathrm{CuO})$ has gained much attention in both fundamental and applied research because of its physical and chemical properties but also due to the great variety of controlled shapes that can be obtained, for example, monolithic or hollow spheres, nanoplates, nanowires, nanobelts, and nanorods. ${ }^{1-3}$ Moreover, as properties of these nanostructured $\mathrm{CuO}$ materials mainly depend on their particle size, orientation, and morphology, great interest in developing novel synthetic pathways for obtaining these nanomaterials exposing different levels of sophistication has arisen in the past few years. ${ }^{4-6}$ It is in this context that we have developed an interest in establishing extremely simple and inexpensive synthetic methodologies to obtain well-defined $\mathrm{CuO}$ nanostructures and demonstrate their application as very efficient catalysts.

Among the most exploited applications of $\mathrm{CuO}$ nanostructures are their uses in catalysis, ${ }^{7}$ sensors, ${ }^{8}$ antibacterial materials, ${ }^{2}$ and environmental remediation. ${ }^{9}$ Moreover, combination of some of these fields has allowed addressing specific cross-topic subjects, for example, the degradation of organic pollutants contained in residual effluents generated by several industries, such as textiles, food, and cosmetics. Understandably, to be useful in this kind of application, the nanostructured $\mathrm{CuO}$ must be considered as a low-cost catalyst, highly efficient, cheap, and if possible reusable and recyclable. There are several colorants often used as model compounds to study the removal of toxic pollutants from water. For instance, methyl orange (MO) has recently been reported to be degraded in aqueous media applying several external stimuli. $^{10-12}$ Different chemical approaches have also been

Received: January 31, 2020

Accepted: February 14, 2020 
reported using from example activators as borohydride ions, ${ }^{13}$ hydrogen peroxide, ${ }^{14,15}$ and a combination of hydrogen peroxide under UV light. ${ }^{16}$ The main disadvantages of these degradation methods are low degradation rates, ${ }^{17}$ use of complicated nanocomposites, ${ }^{10,12,15,16}$ utilization of precious metals as catalysts, ${ }^{13,14}$ and the need for coupling microwave irradiation $^{12}$ or electrochemical systems. ${ }^{12}$ The simplest case for the oxidative degradation of MO might be using hydrogen peroxide as activator (oxidizing agent) and $\mathrm{CuO}$ nanoparticles as the catalytic system. ${ }^{18,19}$ Successful degradation reaction took place at a high cupric oxide nanoleaf $(\mathrm{CuO} \mathrm{NL}) / \mathrm{MO}$ ratio and upon heating. The efficiency of this catalytic system would improve if the surface area of the $\mathrm{CuO}$ nanostructures is increased by simply modifying the aspect ratio or changing the morphology.

The design of synthetic methodologies with the aim of controlling the morphology, crystallinity, and agglomeration of the resulting $\mathrm{CuO}$ nanostructures is a topic of current interest. $^{1,20,21}$ Most of the investigated approaches involve the use of template-assisted hydrothermal reactions, ${ }^{22,23}$ microwave, $^{24,25}$ wet chemical routes, ${ }^{26,27}$ ultrasound, ${ }^{28}$ and electrochemistry. ${ }^{29}$ Among these approaches, wet chemical routes are perhaps the simplest, and precipitation in particular has been recognized as one of the easiest and cheapest yet very effective methods. ${ }^{1}$ For instance, it has been utilized to study the following influences:

- the alkali concentration $(\mathrm{NaOH})$ on the morphology of the nanostructures ${ }^{30}$

- the presence of stabilizer or additive and the preparation temperature on the nanoparticle size and the final morphology ${ }^{31}$

- the volume ratio of the oil-water medium, ${ }^{32}$ the $\mathrm{NaOH}$ temperature, ${ }^{33}$ and the molar ratio $\mathrm{Cu}^{2+} / \mathrm{OH}^{-}$on the size and shape of $\mathrm{CuO}$ nanoparticles

- the use of different precursors $\left(\mathrm{Cu}\left(\mathrm{NO}_{3}\right)_{2} \text { and } \mathrm{CuCl}_{2}\right)^{34}$ and the postheating treatment ${ }^{35}$ on the structural, morphological, and optical properties ${ }^{36}$

- the $\mathrm{pH}$ on the synthesis of $\mathrm{CuO}$ in the presence of polyvinylpyrrolidone ${ }^{37}$

- the concentrations of $\mathrm{CuCl}_{2}$ and $\mathrm{NaOH}$ in a high gravity apparatus $^{38}$

- the aging temperature of the aqueous solution in a Yshaped tubular reactor on the phases and particle size finally obtained and the effect of drying conditions on the morphology 39

Thus, to the best of our knowledge, a comparative study changing the alkali addition conditions using the precipitation method has never been reported hitherto. It was, therefore, the aim of this work to develop two different surfactant-free and low-cost synthetic approaches via the precipitation method to prepare well-defined and highly pure CuO NLs and study their catalytic activity toward the oxidative degradation of MO. The first approach consisted of adding the alkali to a copper solution in a dropwise fashion, thus establishing the DW method. Unlike in the previous case, the second approach involved the addition of the alkali in just one step to the copper solution, establishing the OS method. The morphological, structural, vibrational, and optical properties of the obtained $\mathrm{CuO}$ nanostructures by both methods were studied. Finally, the highly efficient catalytic activity as well as the recyclability of the $\mathrm{CuO}$ nanostructures synthesized in this work toward the oxidative degradation of $\mathrm{MO}$ in the presence of $\mathrm{H}_{2} \mathrm{O}_{2}$ only stimulated by ultrasound and without the influence of light or heat are demonstrated.

\section{EXPERIMENTAL SECTION}

Materials. Copper(II) chloride dihydrate $\left(\mathrm{CuCl}_{2} \cdot 2 \mathrm{H}_{2} \mathrm{O}\right.$, Cicarelli), sodium hydroxide $(\mathrm{NaOH}$, Cicarelli), hydrogen peroxide solution $\left(\mathrm{H}_{2} \mathrm{O}_{2}, 30 \mathrm{wt} \% / \mathrm{wt}\right.$, Anedra Research AG S.A.), and methyl orange (MO, The British Drug House Ltd.) were acquired in analytical grade and used as received.

Synthesis of CuO NLs. Dropwise Method (Hereinafter Abbreviated as DW). To a beaker equipped with a magnetic stirrer containing a $0.1 \mathrm{M} \mathrm{CuCl}_{2} \cdot 2 \mathrm{H}_{2} \mathrm{O}$ solution $(100 \mathrm{~mL})$ was added a 0.1 $\mathrm{M} \mathrm{NaOH}$ solution $(300 \mathrm{~mL})$ in a dropwise fashion (1 drop per second on average) at $30{ }^{\circ} \mathrm{C}$. After $2 \mathrm{~h}$, the reaction mixture was filtered and washed with a large excess of distilled water. The black precipitate obtained was dried until constant weight was reached. Reaction yield: $94 \%$.

One-Step Method (Hereinafter Abbreviated as OS). To a beaker equipped with a magnetic stirrer containing a $0.1 \mathrm{M} \mathrm{CuCl}_{2} \cdot 2 \mathrm{H}_{2} \mathrm{O}$ solution $(100 \mathrm{~mL})$ was added a $0.1 \mathrm{M} \mathrm{NaOH}$ solution $(300 \mathrm{~mL})$ all at once. After $2 \mathrm{~h}$ at $30^{\circ} \mathrm{C}$, the reaction mixture was filtered and washed with a large excess of distilled water. The black precipitate obtained was dried until constant weight was reached. Reaction yield: $96 \%$.

Catalytic Degradation of Methyl Orange. To an aqueous solution of $\mathrm{MO}(10 \mathrm{~mL}, 6.4 \mu \mathrm{g} / \mathrm{mL})$ was added a dispersion of $\mathrm{CuO}$ NLs $(7 \mathrm{~mL}, 53 \mu \mathrm{g} / \mathrm{mL})$ and $\mathrm{H}_{2} \mathrm{O}_{2}(1 \mathrm{~mL}, 30 \% \mathrm{w} / \mathrm{w})$. The reaction system was sonicated for $1 \mathrm{~h}$ at $45 \mathrm{kHz}, 50 \mathrm{~W}$ and then centrifuged for $20 \mathrm{~min}$ at $2000 \mathrm{rpm}$. Different control experiments were conducted to study the possible influence and activity of each component of the reaction system, thus carrying out the same experiment under the same conditions but in presence and absence of $\mathrm{H}_{2} \mathrm{O}_{2}$ or $\mathrm{CuO}$ NLs. Dye degradation was monitored by means of UV-vis spectroscopy in all cases.

Recyclability of the CuO NLs. Once the MO degradation reaction was finished, the catalyst was collected by centrifugation $(2000 \mathrm{rpm})$ at the bottom of the reaction tube. CuO NLs were redispersed within the same reaction tube reestablishing the original reaction conditions described in the previous section (Catalytic Degradation of Methyl Orange) to carry out once again the catalytic degradation of MO. This procedure was repeated to evaluate the recyclability and catalytic efficiency of the recycled $\mathrm{CuO}$ NLs in each of the five consecutive cycles studied. All catalytic experiments were repeated three times obtaining identical results every time.

Characterization Methods. A field emission scanning electron microscope (FESEM, Zeiss Supra 40) with field emission gun operated at $3 \mathrm{kV}$ was employed to determine morphology details of the NLs. Powdered samples were placed on adhesive carbon slice supports. The free version of the software Image $1.51 \mathrm{k}^{40}$ was used to work on the obtained images and build the histograms with data obtained from over 100 measurements analyzing different micrographs for each sample. Crystalline structures, crystallite sizes, and microstrains of $\mathrm{CuO}$ NLs were examined by X-ray diffraction. A SmartLab Rigaku with $\mathrm{Cu} \mathrm{K} \alpha$ radiation in a $2 \theta$ range from $30^{\circ}$ to $90^{\circ}$ in Bragg-Brentano mode and scanning step of $0.02^{\circ}$ at $0.5^{\circ} / \mathrm{min}$ was used. Fourier transform infrared spectra were recorded at room temperature using a FT-IR spectrophotometer, IRAffinity-1 Shimad$\mathrm{zu}$, operating in transmittance mode, accumulating and averaging 60 scans at a resolution of $2 \mathrm{~cm}^{-1}$ in the range of $1200-400 \mathrm{~cm}^{-1}$. Powdered samples were mixed with $\mathrm{KBr}$ in a $1: 83(\mathrm{CuO} / \mathrm{KBr})$ mass ratio. Optical properties of $\mathrm{CuO}$ NLs were studied by UV-vis diffuse reflectance measurements using a Shimadzu UV-2401 PC in the wavelength range of $300-800 \mathrm{~nm}$. Barium sulfate was used as a reference to provide a nominal $100 \%$ reflectance measurement. For these measurements, the powder was milled in a crucible and then dispersed in distilled water to yield a concentration of $74.5 \mu \mathrm{g} / \mathrm{mL}$. The dispersions were sonicated for several minutes until uniform coloration was achieved. 


\section{RESULTS AND DISCUSSION}

Two different synthetic pathways have been design and carried out successfully for the generation of novel low-cost and surfactant-free $\mathrm{CuO}$ NLs. Both strategies are based on the precipitation method but vary the manner in which the alkali was added to the reaction system. Thus, while one synthesis was realized following a dropwise addition of the alkali, the other one was adding the totality of the alkali at once producing a one-step reaction. Both the dropwise (DW) and one-step (OS) methods are presented in Figure 1a. CuO NLs a)

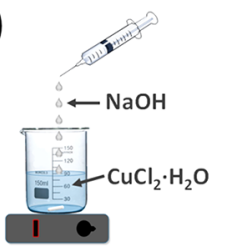

DW method

b)

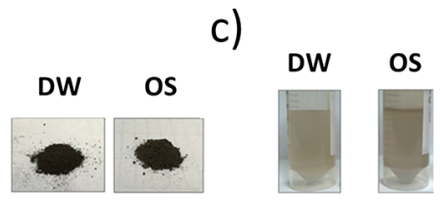

Figure 1. (a) Schematic representation of the dropwise (DW) and one-step (OS) methods for the synthesis of CuO NLs. (b) Pictures of the NL powder obtained following each of the two methods. (c) Pictures of the $\mathrm{CuO}$ NL aqueous dispersions formed using NLs obtained by the DW and OS methods, both at the same concentration of $74.5 \mu \mathrm{g} / \mathrm{mL}$.

were obtained in a powder-like form after filtration, washing, and drying following both the DW and OS methods, as can be seen in Figure 1b. Aqueous dispersions formed using those $\mathrm{CuO}$ NLs are shown in Figure 1c.

The morphological, structural, vibrational, and optical properties of the herein synthesized $\mathrm{CuO}$ NLs as well as their application as recyclable catalyst are presented next.

Field Emission Scanning Electron Microscopy. FESEM was used to investigate the influence of the method in which the basic solution was added on the morphology of the obtained samples. To gain more quantitative information about the morphologies obtained, histograms were constructed for each system by measuring the length $(L)$, width $(W)$, and thickness $(T)$ of the obtained $\mathrm{CuO}$ NLs prepared by both methods, DW and OS. Results are shown in Figure 2.

As can be seen in Figure 2a.1, NLs grown by the DW method agglomerate forming flower-like nanostructures. Practically no NLs were found isolated from the rest. Figure 2a.2,a.3,a.4 are the histograms built showing the frequency distributions for the $\mathrm{CuO} \mathrm{NL}$ length, width, and thickness, respectively. These results showed that the NLs grown by the DW method are on average $281 \mathrm{~nm}$ long, $178 \mathrm{~nm}$ wide, and 17 $\mathrm{nm}$ thick. Figure $2 \mathrm{~b} .1$ reveals that the NLs grown by the OS method are significantly bigger than those obtained by the DW method, being on average $602 \mathrm{~nm}$ long, $219 \mathrm{~nm}$ wide, and 26 $\mathrm{nm}$ thick as determined from the histograms shown in Figure 2b.2,b.3,b.4. A second important difference is that the NLs obtained by the OS method are randomly agglomerated with no predominant geometric form observed. This lack of organization allows for having a better look at their surface, where it was observed that these NLs exhibit rough surfaces.
The dimensions of the $\mathrm{CuO}$ NLs synthesized in the present work are compared in Table 1 with those of other $\mathrm{CuO}$ NLs obtained by a diverse variety of methodologies reported in the literature.

The comparison presented in Table 1 suggests that the sizes of the $\mathrm{CuO}$ NLs strongly depend not only on the synthesis method utilized but also on the surfactant used, if any. The use of stabilizers for synthesizing $\mathrm{CuO}$ nanostructures allows good control of the particle size, particle size distribution, and shape. ${ }^{45}$ However, the high toxicity of some stabilizers, ${ }^{8}$ extra purification steps to remove them afterward, ${ }^{46}$ post-thermal treatments, and centrifugation procedures applied to remove their excess ${ }^{47}$ are time- and resource-consuming processes. Moreover, different stabilizers cause different packing densities on the outer part of crystallites, which may change the crystal growth kinetics and the structural properties of the material. ${ }^{47}$ Also, when nanostructures are passivated chemically, they may expand their lattice constants. ${ }^{48}$ This effect produces larger microstrain distributions making it much tougher to discriminate if the physical or chemical changes are indeed due to the microstrain distribution, the particle size, or the presence of the stabilizer. Based on a different approach, our results demonstrate that using the herein proposed precipitation method for synthesizing $\mathrm{CuO}$ nanostructures leads to the generation of NLs without utilizing any surfactant. The NL sizes obtained were on the same order of magnitude as those using complexing agents. Moreover, the dimensions of the NLs synthesized in this work can be controlled by selecting the right alkali addition methodology. It is recognized in the literature that in general the smaller the nanomaterials are, the higher the agglomeration tendency is, thus trying to reduce the high surface energy. ${ }^{49}$ In fact, this might be the driving force for the $\mathrm{CuO}$ NLs grown by the DW method (smaller size) to exhibit a stronger tendency to agglomerate in a better organized system (flower-like structures) than the ones grown by the OS method (bigger size).

The self-assembly mechanism of CuO NLs is a current topic of discussion. In 2007, $\mathrm{Xu}$ and co-workers ${ }^{50}$ proposed that cupric hydroxide $\left(\mathrm{Cu}(\mathrm{OH})_{2}\right)$ nanoparticles begin a nucleation process, followed by agglomeration forming polycrystalline $\mathrm{Cu}(\mathrm{OH})_{2}$ nanowires, then they agglomerate as $\mathrm{Cu}(\mathrm{OH})_{2}$ NLs. An oriented attachment process between nanowires produces single crystalline $\mathrm{Cu}(\mathrm{OH})_{2}$ NLs, which are finally converted into single crystalline $\mathrm{CuO}$ NLs through a reconstructive transformation. In 2011, Zhao and co-workers ${ }^{51}$ proposed that the $2 \mathrm{D} \mathrm{CuO}$ leaf-like structure formation is from a oriented attachment of $1 \mathrm{D}$ nanorods. These nanorods would originate from different crystallization extents of $\mathrm{CuO}$ assemblies based on the transformation of $\mathrm{Cu}(\mathrm{OH})_{2}$ nanowires in addition to some unspecific interactions with the surfactant. In 2012, Xu and co-workers ${ }^{52}$ reported that $\mathrm{CuO}$ primary crystals directly nucleate from the solution, instead of being converted from $\mathrm{Cu}(\mathrm{OH})_{2}$. The aspect ratio of these primary crystals is intrinsically determined by the surface energies of the (010), (100), and (001) planes. The opposite surfaces of these $\mathrm{CuO}$ primary crystals are oppositely charged and the static attraction force between the positively and negatively charged planes provides the driving force to self-assemble them into NLs. In excess $\mathrm{OH}^{-}$, the much further extended $1 \mathrm{D} \mathrm{CuO}$ primary crystals assemble predominantly on the (100) plane. The selective adsorption of $\mathrm{OH}^{-}$ions on the (001) surfaces fully suppresses assembly on this plane, thus leading to the net $2 \mathrm{D}$ NL morphology. In 2015, Cao and co-workers ${ }^{53}$ proposed that 

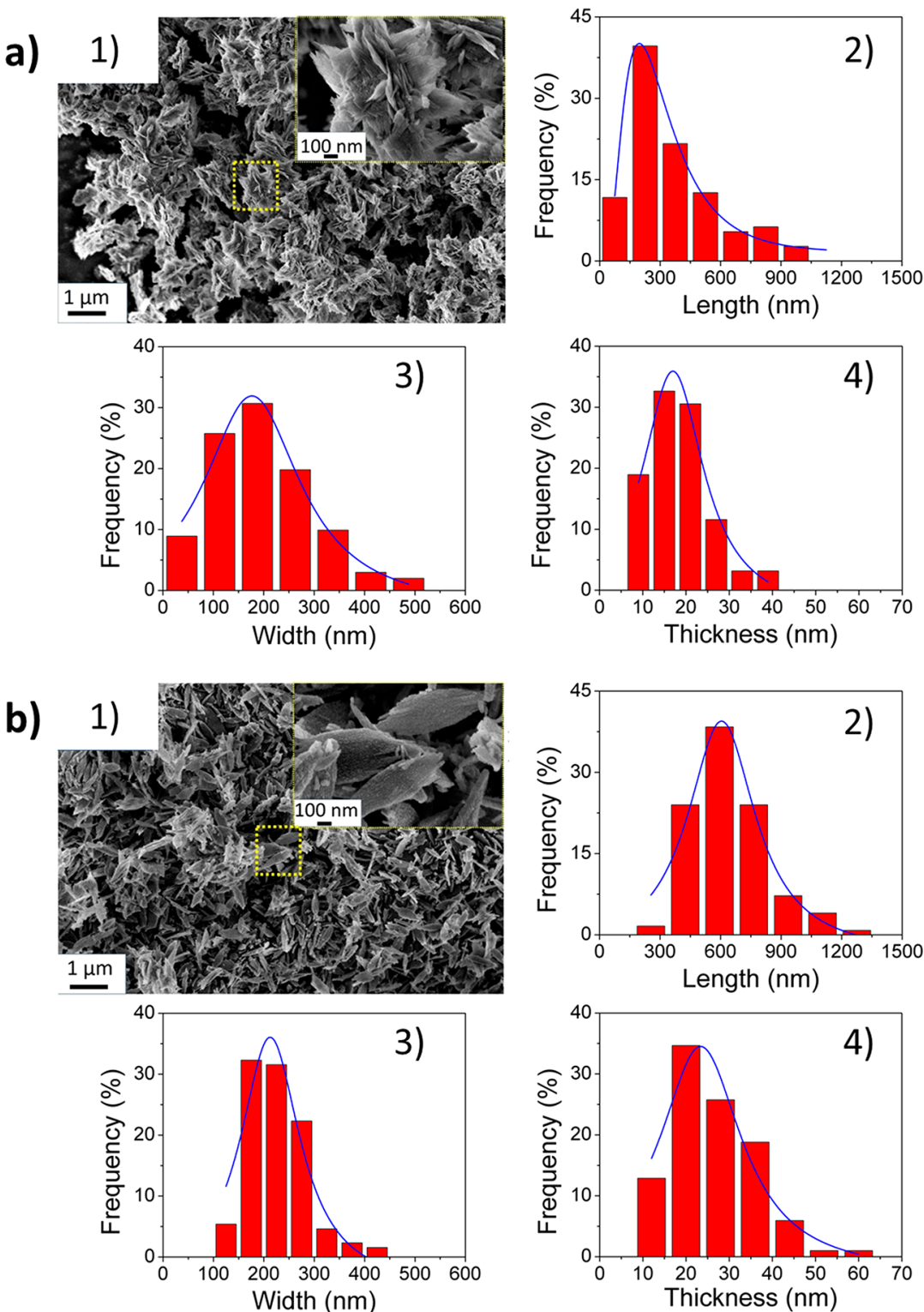

Figure 2. FESEM images of agglomerated CuO NLs obtained by the DW (a.1) and OS (b.1) procedures. The insets show a magnification of a selected area in each picture. Histograms exhibiting the $\mathrm{CuO}$ NL length (a.2 and b.2), width (a.3 and b.3), and thickness (a.4 and b.4) are also shown.

the excess of sodium hydroxide favors the nucleation of $\mathrm{CuO}$ nanocrystals, meanwhile, the decrease of surface energy promotes the oriented attachment of these nanocrystals along the [111] direction into nanowires, finally forming the 2D NLs. More recently, in 2018, Bhattacharjee and Ahmaruzzaman $^{24}$ proposed that $\mathrm{Cu}(\mathrm{OH})_{2}$ nuclei are first formed through a Ostwald ripening process, then the smaller particles agglomerate forming larger particles, which are transformed into $\mathrm{CuO}$. Through self-organization and selfattachment of the $\mathrm{CuO}$ crystals, nanorods are formed. The 2D $\mathrm{CuO} \mathrm{NLs}$ are generated from those one-dimensional $\mathrm{CuO}$ nanorods by an oriented attachment growth mechanism. In addition to the previous examples, there are other authors supporting the hypothesis of the $\mathrm{Cu}(\mathrm{OH})_{2}$ nanowires $^{54}$ or the $\mathrm{CuO}$ nanorods ${ }^{55}$ as fundamental units for the $\mathrm{CuO}$ NLs formation. The two reaction conditions carried out in our work agree with the general and most accepted intermediate that will form the cupric oxide, cupric hydroxide $\left(\mathrm{Cu}(\mathrm{OH})_{2}\right)$. The two alkali addition methodologies utilized generate significant changes in the initial $\mathrm{OH}^{-}$concentration, which might be somehow related to the nucleation environment, playing a crucial role in $\mathrm{CuO}$ NLs formation. Slow $\mathrm{OH}^{-}$ion addition (DW method) favors the formation of small and highly agglomerated NLs, whereas an abrupt increase in the $\mathrm{OH}^{-}$ concentration (OS method) enhances the rates of nucleation and growth, thus allowing the $\mathrm{CuO}$ NLs to be less agglomerated and bigger in size. These observations suggest that changes in the nucleation environment strongly influence the agglomeration process, which in turn affects the sizes of the obtained NLs.

X-ray Diffraction. Figure 3 shows the XRD diffractograms obtained at room temperature of $\mathrm{CuO}$ NLs prepared using both the DW and OS methods. All diffraction peaks in the diffractograms are consistent with the standard data JCPDS PDF card no. 80-1916, indicating the presence of a monoclinic structure of $\mathrm{CuO}$. Moreover, the absence of other diffractions 
Table 1. Summary of Published Results of CuO NLs Dimensions ${ }^{a}$ According to the Synthesis Technique and Stabilizer Employed

\begin{tabular}{|c|c|c|c|c|c|c|}
\hline method & stabilizer & designation & $L^{a}(\mathrm{~nm})$ & $W^{a}(\mathrm{~nm})$ & $T^{a}(\mathrm{~nm})$ & ref \\
\hline \multirow[t]{2}{*}{ precipitation } & - & DW & 281 & 178 & 17 & this study \\
\hline & - & OS & 602 & 219 & 26 & this study \\
\hline \multirow[t]{4}{*}{ hydrothermal } & PEG & - & - & $200-500$ & - & 41 \\
\hline & citrate & - & $50-100$ & - & - & 22 \\
\hline & & & $150-200$ & - & - & \\
\hline & & & $250-300$ & $50-100$ & - & \\
\hline \multirow[t]{5}{*}{ microwave } & L-arginine & - & $350-450$ & $60-90$ & - & 42 \\
\hline & aspartic acid & - & $300-400$ & $50-82$ & - & 25 \\
\hline & glutamic acid & - & $720-800$ & $136-160$ & - & 24 \\
\hline & serine & - & 400 & 86 & - & 43 \\
\hline & sodium dodecyl sulfate & - & $2000-2500$ & $540-660$ & 10 & 44 \\
\hline sonochemical & chitosan & - & $98-171$ & 14 & - & 28 \\
\hline \multirow[t]{2}{*}{ wet chemical } & hexamethylenetetramine & - & $200-363$ & $69-97$ & - & 26 \\
\hline & poly(diallyldimethyl ammonium chloride) & - & 400 & 150 & - & 27 \\
\hline
\end{tabular}

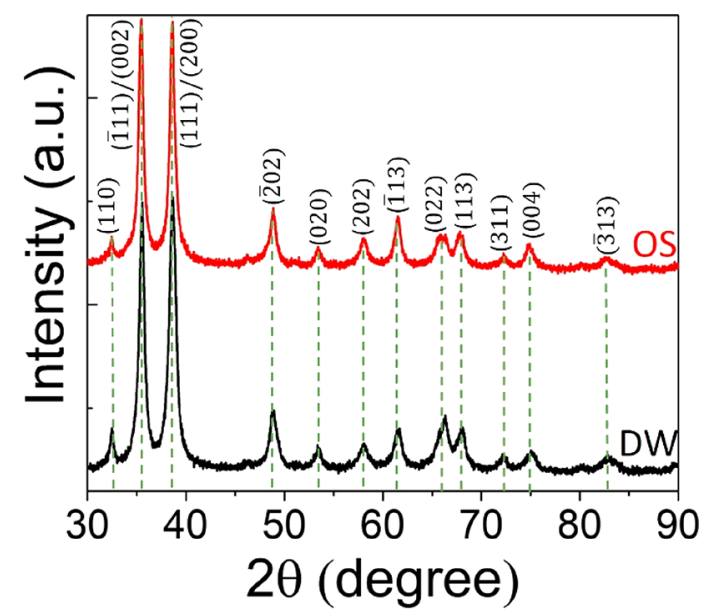

Figure 3. XRD diffractogram patterns observed for the $\mathrm{CuO}$ NLs obtained by the OS and DW methods.

peaks corresponding to cuprite $\left(\mathrm{Cu}_{2} \mathrm{O}\right)$ or cupric hydroxide $\left(\mathrm{Cu}(\mathrm{OH})_{2}\right)$ phases demonstrates that the $\mathrm{CuO}$ NLs could be successfully synthesized without using any surfactant or postsynthesis treatment. Furthermore, these results indicate that the final phase obtained when forming the $\mathrm{CuO} N L s$ is independent of the alkali addition method used in this work.

The average crystallite size $(D)$ and the average local rootmean-square strain $\left(\left\langle\varepsilon_{0}{ }^{2}\right\rangle^{1 / 2}\right)$ were determined using the single-line method. ${ }^{56}$ Results are summarized in Table 2.

It can be seen from the table that the methodology utilized to form the $\mathrm{CuO}$ NLs does not influence the average crystallite size, whereas the alkali addition methodology highly affects the average microstrain distribution. In other words, these results suggest that the nucleation environment, such as the $\mathrm{OH}^{-}$ concentration, plays a crucial role in the microstrain distribution of the resulting NLs. XRD results are also in line with the previously mentioned techniques since the broadening of all peaks in each diffractogram demonstrates the polycrystalline nature of the herein prepared $\mathrm{CuO}$ NLs, each NL being composed of smaller crystallites.

An extra piece of evidence was obtained using the Cohen method, determining the lattice constants of the prepared samples as monoclinic structures. ${ }^{57}$ Results are depicted in Table 2 and are different between them. The differences in lattice parameters might be related to the nanostructure size of the $\mathrm{CuO}$ NLs as well as to the kind of agglomeration. The unit cell volume $(V)$ of DW samples is bigger than that expected for bulk $\mathrm{CuO}\left(81.08 \AA^{3}\right)$, whereas the $V$ value corresponding to OS samples is closer to that for bulk $\mathrm{CuO}{ }^{1}$ The lattice parameters of small isolated nanostructures are often found to contract. $^{58}$ In our case, when the $\mathrm{CuO}$ NLs are freely agglomerated (OS), $V$ is closer to that of the bulk value because of their large dimensions. In contrast, the lattice constants may expand when the nanostructures are passivated chemically or embedded in a matrix. ${ }^{48}$ This might be the case of our flower-like agglomerates (DW). It is worth mentioning that these observations are in full agreement with the microstrain distribution results showed in Table 2. At the same time, $V$ might also be sensitive to the initial $\mathrm{OH}^{-}$ concentration during the $\mathrm{CuO} \mathrm{NL}$ syntheses.

Fourier Transform Infrared Spectroscopy. Figure 4 exhibits the FT-IR spectra of both $\mathrm{CuO}$ NLs obtained following the DW and OS methods. Group theory calculations and experiments have shown that the six IR vibrational bands related to $\mathrm{CuO}$ are located at about $147\left(\mathrm{~B}_{\mathrm{u}}\right), 161\left(\mathrm{~A}_{\mathrm{u}}\right), 321$ $\left(A_{u}\right), 478\left(A_{u}\right), 530\left(B_{u}\right)$, and $590\left(B_{u}\right) \mathrm{cm}^{-1} .59$ Table 3 shows the vibrational bands of the $\mathrm{CuO}$ NLs synthesized in this work (by the DW and OS methods) and those from other CuO NLs reported in the literature for comparison.

Table 2. Average Crystallite Size $(D)$ and Average Local Root-Mean-Square Strain $\left(\left\langle\varepsilon_{0}{ }^{2}\right\rangle^{1 / 2}\right)$, Lattice Parameters $(a, b, c$, and $\beta)$, and Cell Unit Volume $(V)$ of $\mathrm{CuO}$ Obtained by the DW and OS Methods ${ }^{a}$

$\begin{array}{cccccccc}\text { sample } & D(\mathrm{~nm}) & \left\langle\varepsilon_{0}^{2}\right\rangle^{1 / 2} & a(\AA) & b(\AA) & c(\AA) & \beta(\operatorname{deg}) & V\left(\AA^{3}\right) \\ \text { DW } & 16(6) & 0.0107(2) & 4.688(1) & 3.431(1) & 5.126(1) & 99.305(1) & 81.4(1) \\ \text { OS } & 16(5) & 0.0078(1) & 4.675(2) & 3.418(2) & 5.132(2) & 99.171(2) & 81.0(1)\end{array}$

${ }^{a}$ Uncertainty is expressed in parentheses. 


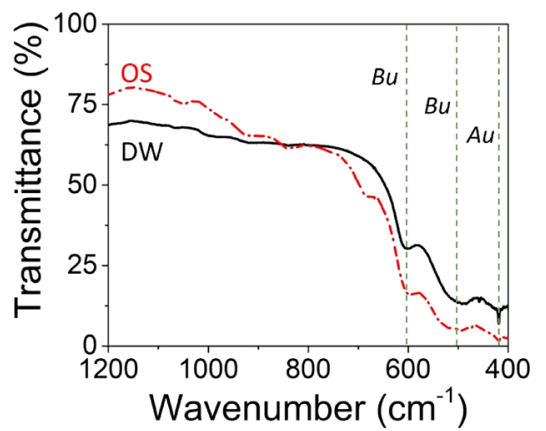

Figure 4. FT-IR spectra of both $\mathrm{CuO}$ NLs obtained following the DW (-) and OS (-.- ) methods.

The two high frequency modes observed in Figure 4 at 609 and $513 \mathrm{~cm}^{-1}$ for DW and at 603 and $527 \mathrm{~cm}^{-1}$ for OS are assigned to the $\mathrm{Cu}-\mathrm{O}$ stretching vibration along the [202] direction, while those observed at $419 \mathrm{~cm}^{-1}$ for DW and at 421 $\mathrm{cm}^{-1}$ for $\mathrm{OS}$ are assigned to the $\mathrm{Cu}-\mathrm{O}$ stretching vibration along the [202] direction. These results are in agreement with those previously reported. ${ }^{61}$ It is worth mentioning at this point that the absence of vibrational bands at about 694,700 , 820 , and $934 \mathrm{~cm}^{-1}$, characteristics of $\mathrm{Cu}_{2} \mathrm{O}^{62,63}$ and $\mathrm{Cu}-$ $(\mathrm{OH})_{2},{ }^{61,64}$ evidences the high purity of the $\mathrm{CuO}$ NLs. This result is in agreement with the previously discussed XRD results. The differences observed in the peak positions suggest that the vibrational modes might be sensitive to both the crystallites sizes and the internal strain. These differences are more important for $\mathrm{CuO}$ NLs obtained using surfactants (entries 3-10, Table 3). It has been reported that the addition of complexing agents in the synthesis generates nonuniform microstrains in $\mathrm{CuO} .^{65}$

Optical Absorption. The type of electronic transition in $\mathrm{CuO}$ nanostructures is a topic of current discussion in the literature. ${ }^{66}$ The indirect transition is much less reported in the literature ${ }^{65}$ than the direct transition, ${ }^{67}$ which appears to be the predominant phenomenon. Thus, we developed an interest in obtaining band gap energy $\left(E_{g}\right)$ values from both indirect and direct transitions. To achieve this, the Kubelka-Munk remission function (converted from the diffuse reflection values) was obtained. ${ }^{65}$ The indirect band gap energies of the $\mathrm{CuO}$ NLs synthesized in this work were determined from the plot of $[F(R) h \nu]^{1 / 2}$ as a function of the energy $(\mathrm{eV})$, where the function $F(R)$ is the equivalent to the absorption coefficient $(\alpha)$. The extrapolation of the linear region for each system (DW and OS) until it intersects the abscissa, the energy axis, reveals the band gap energy value (as shown in Figure 5a).
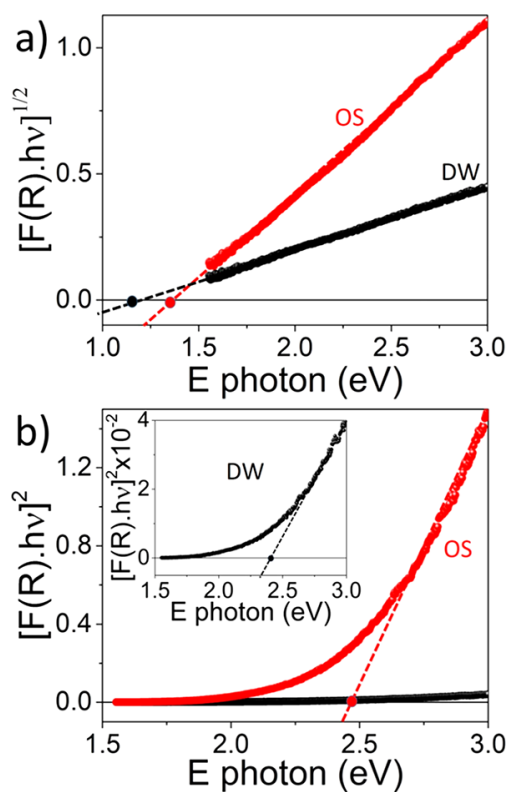

Figure 5. Plots of $[F(R) h \nu]^{1 / 2}$ and $[F(R) h \nu]^{2}$ as a function of the energy for both an indirect electronic transition (a) and a direct electronic transition (b). The inset in panel $\mathrm{b}$ is a magnification of the region between 1.5 and $3.0 \mathrm{eV}$ and 0.00 and $0.04[F(R) h \nu]^{2}$ from the plot obtained for DW samples.

Thus, indirect band gap energy values of $1.21 \pm 0.01$ and 1.39 $\pm 0.01 \mathrm{eV}$ are determined for CuO NLs obtained by the DW and OS methods, respectively. These values are in agreement with those expected for $\mathrm{CuO}$ in bulk $(1.2 \mathrm{eV}){ }^{1}$

Figure $5 \mathrm{~b}$ shows $[F(R) h \nu]^{2}$ as a function of the energy for samples of $\mathrm{CuO}$ NLs synthesized in this work. Once again, the interception between the extrapolated linear region of the graph and the abscissa corresponds to the band gap energy of the $\mathrm{CuO}$ NLs herein obtained, although this time is for a direct

Table 3. Summary of Vibrational Modes and Direct Band Gap Energies Related to the CuO NLs Synthesized Following the DW and OS Methods Compared to Those from the Literature When Using Stabilizers

\begin{tabular}{|c|c|c|c|c|c|c|}
\hline \multirow[b]{2}{*}{ entry } & \multicolumn{6}{|c|}{ vibrational modes $\left(\mathrm{cm}^{-1}\right)$} \\
\hline & system or stabilizer & $\mathrm{A}_{\mathrm{u}}$ & $\mathrm{B}_{\mathrm{u}}$ & $\mathrm{B}_{\mathrm{u}}$ & direct band gap energy $(\mathrm{eV})$ & ref \\
\hline 1 & DW & 419 & 513 & 609 & $2.38 \pm 0.05$ & this work \\
\hline 2 & OS & 421 & 527 & 603 & $2.46 \pm 0.05$ & this work \\
\hline 3 & PEG & 440 & 564 & 600 and 628 & - & 41 \\
\hline 4 & poly(diallyldimethyl ammonium chloride) & 423 & 498 & 608 & 1.98 & 60 \\
\hline 5 & chitosan & 434 & 514 & 611 & - & 28 \\
\hline 6 & L-arginine & 418 & 498 & 610 & 2.15 & 42 \\
\hline 7 & aspartic acid & 420 & 546 & 600 & 2.20 & 25 \\
\hline 8 & glutamic acid & 420 & 511 & 600 & 2.10 & 24 \\
\hline 9 & L-serine & 426 & 546 & 597 & 2.30 & 43 \\
\hline 10 & hydrazine hydrate & - & - & - & 2.17 & 53 \\
\hline 11 & stabilizer free & - & - & - & 2.67 & 22 \\
\hline 12 & trisodium citrate & - & - & - & 2.97 & 22 \\
\hline 13 & sodium citrate & - & - & - & 2.80 & 22 \\
\hline 14 & sodium dodecyl sulfate & - & - & - & 2.13 & 44 \\
\hline
\end{tabular}


transition. These results are shown in Table 3 and compared to those from the literature. It is important to highlight that our results agree with those already reported as shown in the table.

Most authors indicate that the blue shift in the band gap energy observed in $\mathrm{CuO}$ NLs is due to quantum confinement effects. It must be mentioned that in almost all those reports the $\mathrm{CuO}$ NLs have both their lengths and widths greater than the Bohr radius (from 6.6 to $28.7 \mathrm{~nm}$ ) ${ }^{1}$ where the quantum effects are important. We consider that the NL thickness is the dimension in which quantum effects might arise the most and should no longer be neglected, allowing for deeper understanding the differences found in those reported band gap energies. As shown in Table 1, unfortunately, the thickness of $\mathrm{CuO}$ NLs is the least reported dimension in the literature for this kind of material. We believe that the blue shift in the band gap energy with respect to the bulk value is too big for one dimension in the quantum confinement regime. For that reason, in our opinion, the band gap energy values could be overestimated when it is considered to be a direct transition. These observations are in agreement with the theoretical calculation results obtained from the electronic band structure of a series of different cupric oxides. ${ }^{68}$ In the case of an indirect electronic transition, the blue shift observed for the herein synthesized $\mathrm{CuO}$ NLs might be due to a combination of the NL thickness, general size of the crystallites, and the microstrain distribution, in accordance with the results discussed in the previous sections. Nevertheless, more sophisticated studies must be carried out in order to gain clearer insights in this direction.

Catalytic Activity and Recyclability of CuO NLs. From an applied nanomaterials science standpoint, it was easy to envision these $\mathrm{CuO}$ NLs as effective catalysts. Thus, with the interest of evaluating the catalytic activity of the CuO NLs synthesized in this work, the oxidative degradation of a model dye, methyl orange (hereinafter abbreviated as MO), was studied. To achieve this goal, a set of complementary experiments were carried out realizing the same chemical reaction in darkness (thus avoiding photocatalysis), in the presence and absence of an oxidizing agent (hydrogen peroxide, $\mathrm{H}_{2} \mathrm{O}_{2}$ ), with and without the catalyst ( $\mathrm{CuO}$ NLs), with no extra heating, assisted by ultrasound, and followed by the same workup. Figure 6a shows a summary of the main results obtained after carrying out every control experiment and the actual oxidative degradation reaction under the mentioned conditions, which are detailed in the Experimental Section.

MO presented the very same strong absorption band with maximum at $464 \mathrm{~nm}$ before and after the sonication and centrifugation steps; the UV-vis absorption spectrum is shown in Figure 6a.i. When the same MO aqueous solution was treated in the presence of $\mathrm{CuO}$ NLs obtained by the OS method, the intensity of the absorption band is very slightly reduced, corresponding to a concentration decrease less than $1 \%$ (Figure 6a.ii). Addition of $\mathrm{H}_{2} \mathrm{O}_{2}$ to the $\mathrm{MO}$ aqueous solution produced degradation of the dye as can be observed by the decreased absorbance at $464 \mathrm{~nm}$ (Figure 6a.iii), corresponding to a concentration reduction of about $14 \%$. It is worth mentioning that the $\mathrm{H}_{2} \mathrm{O}_{2}$ aqueous solution presents a strong absorption below $350 \mathrm{~nm}$ with the tail of that peak weakly interfering within the region of interest (Figure 6a.iv). Finally, a nearly complete disappearance of the absorption band at $464 \mathrm{~nm}$ corresponding to the dye is seen in Figure 6a.v upon measuring the reaction product obtained from the system
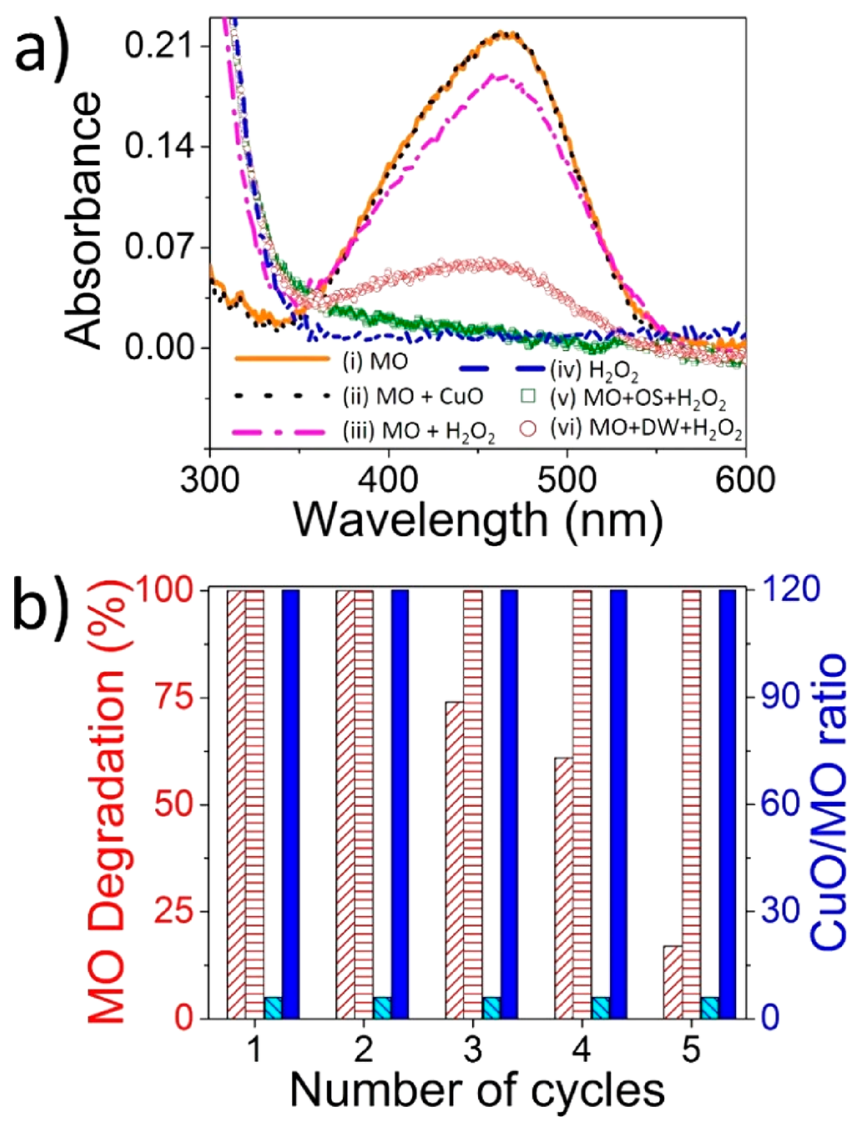

Figure 6. (a) UV-vis absorption spectra of each resulting system after having carried out the catalytic oxidative degradation of $\mathrm{MO}$ and all control experiments. (b) Evaluation of the efficiency of the catalytic oxidative degradation reaction of $\mathrm{MO}$ using $\mathrm{CuO}$ NLs obtained by the OS method as a function of the number of times it is recycled (red diagonal striped bars) in comparison with data reported from the literature for $\mathrm{CuO}$ nanoparticles (red horizontal striped bars). ${ }^{18}$ The graph also shows the catalyst/dye ratio (CuO NLs/MO (cyan bars) versus $\mathrm{CuO}$ nanoparticles/MO (blue bars) $)^{18}$ by weight used for each of the five times the catalysts were recycled.

formed by mixing the $\mathrm{MO}$ aqueous solution, $\mathrm{H}_{2} \mathrm{O}_{2}$, and the $\mathrm{CuO}$ NLs synthesized by the OS method.

These results indicate that $\mathrm{MO}$ is stable under the sonication and centrifugation conditions carried out in darkness independently of the presence or absence of the $\mathrm{CuO}$ NLs synthesized by the OS method used as catalyst. However, the oxidizing agent is active under the reaction conditions even in absence of the catalyst, degrading a maximum of $14 \%$ of the dye. It has been reported that sonication promotes chemical reactions by acoustic cavitation, which forms minute bubbles that implode thus generating very high localized temperatures and pressures. ${ }^{69}$ Under these conditions, $\mathrm{H}_{2} \mathrm{O}_{2}$ can easily generate hydroxyl radicals, which have been recognized as the main factor for dye decomposition. ${ }^{70}$ This explains why MO is partially degraded by $\mathrm{H}_{2} \mathrm{O}_{2}$ in absence of the catalyst under the reaction conditions used in this work while it seems to be relatively stable under mechanical stirring and thermal heating. ${ }^{18}$ The most relevant result is related to the complete disappearance of the absorption band at $464 \mathrm{~nm}$, which strongly suggests that MO was $100 \%$ chemically degraded through a catalytic oxidative reaction carried out at room temperature, promoted by $\mathrm{H}_{2} \mathrm{O}_{2}$, and very efficiently catalyzed by the $\mathrm{CuO}$ NLs obtained using the OS method. In the case of 
the $\mathrm{CuO}$ NLs synthesized by the DW method, the overall results are similar to the ones obtained for the $\mathrm{CuO}$ NLs obtained by the OS method, except with less efficient catalytic activity (73\%) (Figure 6a.vi). The difference in the catalytic efficiency between the two $\mathrm{CuO}$ NLs systems can be rationalized as an adequate balance involving the surface area, agglomeration, and dispersibility. In addition to these variables, which might be studied deeply, other parameters must be further investigated such as $\mathrm{pH}$, temperature, agitation or stirring method, and relative concentration of reactants. These studies exceed the scope of the present work, although they are currently underway and will be reported opportunely.

It is worth mentioning that a similar reaction system has been reported by Deka and co-workers ${ }^{18}$ claiming successful catalytic activity of $\mathrm{CuO}$ nanostructures in the presence of $\mathrm{H}_{2} \mathrm{O}_{2}$ for reactions carried out utilizing a high catalyst dosage (60:1 catalyst/MO by weight) at $65{ }^{\circ} \mathrm{C}$. Therefore, the three reaction systems, the reported $\mathrm{CuO}$ nanostructures and the $\mathrm{CuO}$ NLs synthesized by the OS and DW methods in this work, can be considered as successful. However, the system formed using the $\mathrm{CuO}$ NLs grown by the OS method might be seen as the most efficient since it can be realized without a thermal treatment and utilizing a much lower catalyst dosage (about 6:1 catalyst/MO by weight). This difference in favor of the $\mathrm{CuO}$ NLs obtained by the OS method in their catalytic activity might be related to the better aspect ratio of the NLs compared to nanoparticles because of the higher surface area exposed, which has been reported to be one of the main contributors for enhancing the catalytic efficiency. ${ }^{23}$

The report presented by Deka and co-workers ${ }^{18}$ also presents an evaluation of the recyclability of the $\mathrm{CuO}$ nanoparticles synthesized, showing an extremely high efficiency of the catalyst toward the degradation of $\mathrm{MO}$ in the five cycles studied, but using for this test a catalyst/MO ratio of 120:1 by weight (Figure 6b). This study in addition to our also highly efficient catalysts further motivated us to explore the recyclability of our best catalyst, $\mathrm{CuO}$ NLs obtained by the OS method. To achieve this goal, we designed a set of experiments where the $\mathrm{CuO}$ NLs that were separated by centrifugation as part of the workup once the degradation reaction was finished are reutilized as the catalyst for a new degradation reaction. This new reaction is carried out under identical reaction conditions as the previous one. This was feasible because of the good dispersibility of the used $\mathrm{CuO}$ NLs upon addition of the rest of the reactants and solvents to the reaction flask. This procedure was repeated several times and the efficiency of the degradation reaction as a function of the number of cycles carried out is shown in Figure $6 \mathrm{~b}$. As can be seen in the figure, the efficiency of the degradation reaction of $\mathrm{MO}$ under the catalytic oxidative conditions is highly efficient, achieving a reaction yield of $100 \%$ for the two first cycles. From this point forward, however, the degradation reaction yield obtained after each consecutive cycle started to drop to $74 \%, 61 \%$, and $17 \%$ for the third, fourth, and fifth cycles, respectively. This last efficiency is, in fact, comparable to that of a system formed by the aqueous solution of $\mathrm{MO}$ in the presence of $\mathrm{H}_{2} \mathrm{O}_{2}$ but in absence of $\mathrm{CuO}$ NLs (Figure 6a). It must be emphasized at this point that the recyclability study carried out using the herein synthesized $\mathrm{CuO}$ NLs was realized using a catalyst/MO ratio of $6: 1$ by weight, in other words 20 times smaller than that of the previously reported system (Figure 6b). ${ }^{18}$ Moreover, an enhancement is achieved not only in terms of the amount of catalyst needed but also by the fact that the herein presented system works perfectly without any extra thermal treatment.

\section{CONCLUSIONS}

High purity copper oxide (II) polycrystalline nanoleaves $(\mathrm{CuO}$ NLs) composed of small crystallites were successfully synthesized carrying out a simple and low-cost precipitation technique. The obtained $\mathrm{CuO}$ NLs were similar in size to those synthesized using complexing agents. The effects of the nucleation environment (alkali addition method) on the morphological, structural, vibrational, and optical properties of the resulting NLs were studied. With the DW method, $\mathrm{CuO}$ NLs agglomerated forming flower-like structures, which are not easily dispersed in water. Thus, these NLs presented low catalytic activity toward the oxidative degradation of MO. In contrast, with the OS method, bigger CuO NLs were obtained, which showed random agglomeration. These NLs presented better catalytic activity than the ones obtained by the DW method. Concerning the band gap energy studies, our results suggest that an electronic indirect transition might be more feasible than a direct one. This last result might be complemented in the future performing more sophisticated studies in order to gain clearer insights in this direction. Moreover, the applicability of these $\mathrm{CuO}$ NLs as highly efficient and recyclable catalysts was demonstrated by studying the catalytic oxidative degradation of methyl orange in the presence of an oxidizing agent, $\mathrm{H}_{2} \mathrm{O}_{2}$, stimulated by ultrasound and without any thermal treatment. Finally, we expect our results to contribute from an environmental remediation standpoint since these catalytic systems might be useful for treating industrial aqueous effluents.

\section{AUTHOR INFORMATION}

\section{Corresponding Authors}

Nora J. François - Applications of Biocompatible Materials Group, Chemistry Department, School of Engineering and Institute of Technology in Polymers and Nanotechnology (ITPN), UBA-CONICET, University of Buenos Aires, Buenos Aires C1063ACV, Argentina; Email: nfranco@fi.uba.ar

Pablo Froimowicz - Design and Chemistry of Macromolecules Group, Institute of Technology in Polymers and Nanotechnology (ITPN), UBA-CONICET, FADU, University of Buenos Aires, Buenos Aires C1428EGA, Argentina; (1) orcid.org/0000-00031154-1024; Email: pxf106@case.edu

\section{Authors}

Cesar L. Londoño-Calderón - Design and Chemistry of Macromolecules Group, Institute of Technology in Polymers and Nanotechnology (ITPN), UBA-CONICET, FADU, University of Buenos Aires, Buenos Aires C1428EGA, Argentina

Sandra Menchaca-Nal - Design and Chemistry of Macromolecules Group, Institute of Technology in Polymers and Nanotechnology (ITPN), UBA-CONICET, FADU and Applications of Biocompatible Materials Group, Chemistry Department, School of Engineering, University of Buenos Aires, Buenos Aires C1428EGA, Argentina

Laura G. Pampillo - Institute of Technology and Engineering Sciences "Hilario Fernández Long" (INTECIN-CONICET), School of Engineering, University of Buenos Aires, Buenos Aires C1063ACV, Argentina

Complete contact information is available at: https://pubs.acs.org/10.1021/acsanm.0c00283 


\section{Notes}

The authors declare no competing financial interest.

\section{ACKNOWLEDGMENTS}

Financial support from Agencia Nacional de Promoción Científica y Tecnológica, ANPCyT (Préstamo BID through projects PICT 2014-1955 and 2016-0843), and Consejo Nacional de Investigaciones Cientificas y Técnicas, CONICET (PUE 22920160100034CO and PUE 22920170100073CO), is gratefully acknowledged.

\section{REFERENCES}

(1) Zhang, Q.; Zhang, K.; Xu, D.; Yang, G.; Huang, H.; Nie, F.; Liu, C.; Yang, S. CuO Nanostructures: Synthesis, Characterization, Growth Mechanisms, Fundamental Properties, and Applications. Prog. Mater. Sci. 2014, 60 (1), 208-337.

(2) Karim, M. N.; Singh, M.; Weerathunge, P.; Bian, P.; Zheng, R.; Dekiwadia, C.; Ahmed, T.; Walia, S.; Della Gaspera, E.; Singh, S.; Ramanathan, R.; Bansal, V. Visible-Light-Triggered Reactive-OxygenSpecies-Mediated Antibacterial Activity of Peroxidase-Mimic $\mathrm{CuO}$ Nanorods. ACS Applied Nano Materials 2018, 1 (4), 1694-1704.

(3) Su, Y.; Li, G.; Guo, Z.; Li, Y.-Y.; Li, Y.-X.; Huang, X.-J.; Liu, J.-H. Cation-Exchange Synthesis of Cu2Se Nanobelts and Thermal Conversion to Porous $\mathrm{CuO}$ Nanobelts with Highly Selective Sensing toward H2S. ACS Applied Nano Materials 2018, 1 (1), 245-253.

(4) Ali, G.; Park, Y. J.; Kim, J. W.; Cho, S. O. A Green, General, and Ultrafast Route for the Synthesis of Diverse Metal Oxide Nanoparticles with Controllable Sizes and Enhanced Catalytic Activity. ACS Applied Nano Materials 2018, 1 (11), 6112-6122.

(5) Karunakaran, C.; Manikandan, G.; Gomathisankar, P. Microwave, Sonochemical and Combustion Synthesized $\mathrm{CuO}$ Nanostructures and Their Electrical and Bactericidal Properties. J. Alloys Compd. 2013, 580, 570-577.

(6) Sadollahkhani, A.; Hussain Ibupoto, Z.; Elhag, S.; Nur, O.; Willander, M. Photocatalytic Properties of Different Morphologies of $\mathrm{CuO}$ for the Degradation of Congo Red Organic Dye. Ceram. Int. 2014, 40 (7), 11311-11317.

(7) Wang, L.; Hou, J.; Liu, S.; Carrier, A. J.; Guo, T.; Liang, Q.; Oakley, D.; Zhang, X. CuO Nanoparticles as Haloperoxidase-Mimics: Chloride-Accelerated Heterogeneous Cu-Fenton Chemistry for H2O2 and Glucose Sensing. Sens. Actuators, B 2019, 287, 180-184.

(8) Kamali, M.; Samari, F.; Sedaghati, F. Low-Temperature PhytoSynthesis of Copper Oxide Nanosheets: Its Catalytic Effect and Application for Colorimetric Sensing. Mater. Sci. Eng., C 2019, 103, 109744.

(9) Egirani, D.; Latif, M. T.; Wessey, N.; Poyi, N. R.; Acharjee, S. Synthesis and Characterization of Kaolinite Coated with Copper Oxide and Its Effect on the Removal of Aqueous Lead(II) Ions. Appl. Water Sci. 2019, 9 (4), 109.

(10) Zhao, Q.; Wang, K.; Wang, J.; Guo, Y.; Yoshida, A.; Abudula, A.; Guan, G. Cu2O Nanoparticle Hyper-Cross-Linked Polymer Composites for the Visible-Light Photocatalytic Degradation of Methyl Orange. ACS Applied Nano Materials 2019, 2 (5), 27062712.

(11) Wang, Y.; Gai, L.; Ma, W.; Jiang, H.; Peng, X.; Zhao, L. Ultrasound-Assisted Catalytic Degradation of Methyl Orange with Fe3O4/Polyaniline in Near Neutral Solution. Ind. Eng. Chem. Res. 2015, 54 (8), 2279-2289.

(12) Bai, D.; Yan, P. Magnetic Nanoscaled Fe3O4 as an Efficient and Reusable Heterogeneous Catalyst for Degradation of Methyl Orange in Microwave-Enhanced Fenton-Like System. Appl. Mech. Mater. 2013, 448-453, 830-833.

(13) Sakir, M.; Onses, M. S. Solid Substrates Decorated with Ag Nanostructures for the Catalytic Degradation of Methyl Orange. Results Phys. 2019, 12, 1133-1141.

(14) Gao, Q.; Xing, Y.; Peng, M.; Liu, Y.; Luo, Z.; Jin, Y.; Fan, H.; Li, K.; Chen, C.; Cui, Y. Enhancement of $\mathrm{Fe} 3 \mathrm{O} 4 / \mathrm{Au}$ Composite
Nanoparticles Catalyst in Oxidative Degradation of Methyl Orange Based on Synergistic Effect. Chin. J. Chem. 2017, 35 (9), 1431-143. (15) Xu, H. Y.; Wang, Y.; Shi, T. N.; Zhao, H.; Tan, Q.; Zhao, B. C.; He, X. L.; Qi, S. Y. Heterogeneous Fenton-Like Discoloration of Methyl Orange Using Fe3O4/MWCNTs as Catalyst: Kinetics and Fenton-Like Mechanism. Frontiers of Materials Science 2018, 12 (1), 34-44.

(16) Arshad, A.; Iqbal, J.; Ahmad, I.; Israr, M. Graphene/Fe3O4 Nanocomposite: Interplay Between Photo-Fenton Type Reaction, and Carbon Purity for the Removal of Methyl Orange. Ceram. Int. 2018, 44 (3), 2643-2648.

(17) Devi, H. S.; Singh, T. D. Synthesis of Copper Oxide Nanoparticles by a Novel Method and its Application in the Degradation of Methyl Orange. Advance in Electronic and Electric Engineering 2014, 4 (1), 83-88.

(18) Deka, P.; Hazarika, A.; Deka, R. C.; Bharali, P. Influence of $\mathrm{CuO}$ Morphology on the Enhanced Catalytic Degradation of Methylene Blue and Methyl Orange. RSC Adv. 2016, 6 (97), 95292-95305.

(19) Deka, P.; Deka, R. C.; Bharali, P. Porous CuO Nanostructure as a Reusable Catalyst for Oxidative Degradation of Organic Water Pollutants. New J. Chem. 2016, 40 (1), 348-357.

(20) Moniri Javadhesari, S.; Alipour, S.; Mohammadnejad, S.; Akbarpour, M. R. Antibacterial Activity of Ultra-Small Copper Oxide (II) Nanoparticles Synthesized by Mechanochemical Processing Against S. Aureus and E. Coli. Mater. Sci. Eng., C 2019, 105, 110011.

(21) Chauhan, M.; Sharma, B.; Kumar, R.; Chaudhary, G. R.; Hassan, A. A.; Kumar, S. Green Synthesis of $\mathrm{CuO}$ Nanomaterials and Their Proficient Use for Organic Waste Removal and Antimicrobial Application. Environ. Res. 2019, 168, 85-95.

(22) Sonia, S.; Poongodi, S.; Kumar, P. S.; Mangalaraj, D.; Ponpandian, N.; Viswanathan, C. Hydrothermal Synthesis of Highly Stable $\mathrm{CuO}$ Nanostructures for Efficient Photocatalytic Degradation of Organic Dyes. Mater. Sci. Semicond. Process. 2015, 30, 585-591.

(23) Liu, J.; Jin, J.; Deng, Z.; Huang, S.-Z.; Hu, Z.-Y.; Wang, L.; Wang, C.; Chen, L.-H.; Li, Y.; Van Tendeloo, G.; Su, B.-L. Tailoring $\mathrm{CuO}$ Nanostructures for Enhanced Photocatalytic Property. J. Colloid Interface Sci. 2012, 384 (1), 1-9.

(24) Bhattacharjee, A.; Ahmaruzzaman, M. Microwave Assisted Facile and Green Route for Synthesis of $\mathrm{CuO}$ Nanoleaves and Their Efficacy as a Catalyst for Reduction and Degradation of Hazardous Organic Compounds. J. Photochem. Photobiol., A 2018, 353, 215-228.

(25) Bhattacharjee, A.; Begum, S.; Neog, K.; Ahmaruzzaman, M. Facile Synthesis of 2D CuO Nanoleaves for the Catalytic Elimination of Hazardous and Toxic Dyes from Aqueous Phase: a Sustainable Approach. Environ. Sci. Pollut. Res. 2016, 23 (12), 11668-11676.

(26) Xia, Y.; Pu, X.; Liu, J.; Liang, J.; Liu, P.; Li, X.; Yu, X. CuO Nanoleaves Enhance the c-Si Solar Cell Efficiency. J. Mater. Chem. A 2014, 2 (19), 6796-6800.

(27) Manoj, D.; Ranjith Kumar, D.; Santhanalakshmi, J. Impact of $\mathrm{CuO}$ Nanoleaves on MWCNTs/GCE Nanocomposite Film Modified Electrode for the Electrochemical Oxidation of Folic Acid. Appl. Nanosci. 2012, 2 (3), 223-230.

(28) Abiraman, T.; Ramanathan, E.; Kavitha, G.; Rengasamy, R.; Balasubramanian, S. Synthesis of Chitosan Capped Copper Oxide Nanoleaves Using High Intensity $(30 \mathrm{kHz})$ Ultrasound Sonication and Their Application in Antifouling Coatings. Ultrason. Sonochem. 2017, 34, 781-791.

(29) Joya, K. S.; de Groot, H. J. M. Controlled Surface-Assembly of Nanoscale Leaf-Type Cu-Oxide Electrocatalyst for High Activity Water Oxidation. ACS Catal. 2016, 6 (3), 1768-1771.

(30) Singh, D. P.; Ojha, A. K.; Srivastava, O. N. Synthesis of Different $\mathrm{Cu}(\mathrm{OH}) 2$ and $\mathrm{CuO}$ (Nanowires, Rectangles, Seed-, Belt-, and Sheetlike) Nanostructures by Simple Wet Chemical Route. J. Phys. Chem. C 2009, 113 (9), 3409-3418.

(31) Zhu, J.; Li, D.; Chen, H.; Yang, X.; Lu, L.; Wang, X. Highly Dispersed $\mathrm{CuO}$ Nanoparticles Prepared by a Novel QuickPrecipitation Method. Mater. Lett. 2004, 58 (26), 3324-3327. 
(32) Wu, R.; Ma, Z.; Gu, Z.; Yang, Y. Preparation and Characterization of $\mathrm{CuO}$ Nanoparticles with Different Morphology through a Simple Quick-Precipitation Method in DMAC-Water Mixed Solvent. J. Alloys Compd. 2010, 504 (1), 45-49.

(33) Zhu, J.; Bi, H.; Wang, Y.; Wang, X.; Yang, X.; Lu, L. CuO Nanocrystals with Controllable Shapes Grown from Solution without any Surfactants. Mater. Chem. Phys. 2008, 109 (1), 34-38.

(34) Phiwdang, K.; Suphankij, S.; Mekprasart, W.; Pecharapa, W. Synthesis of $\mathrm{CuO}$ Nanoparticles by Precipitation Method Using Different Precursors. Energy Procedia 2013, 34, 740-745.

(35) Lin, C. C.; Wu, M. S. Continuous Production of $\mathrm{CuO}$ Nanoparticles in a Rotating Packed Bed. Ceram. Int. 2016, 42 (2), 2133-2139.

(36) Siddiqui, H.; Qureshi, M. S.; Haque, F. Z. Effect of Copper Precursor Salts: Facile and Sustainable Synthesis of Controlled Shaped Copper Oxide Nanoparticles. Optik 2016, 127 (11), 47264730

(37) Shahmiri, M.; Ibrahim, N. A.; Zainuddin, N.; Asim, N.; Bakhtyar, B.; Zaharim, A.; Sopian, K. Effect of $\mathrm{pH}$ on the Synthesis of Cuo Nanosheets by Quick Precipitation Method. WSEAS Transactions on Environment and Development 2013, 9 (2), 137-145.

(38) Chen, P. C.; Lin, J. Effect of Solution Condition on the Precipitation of Nano-Cupric Oxide by Using a High Gravity Process. J. Taiwan Inst. Chem. Eng. 2016, 59, 514-520.

(39) Kameyama, N.; Senna, M. Effects of Aging Temperature on the Size and Morphology of $\mathrm{Cu}(\mathrm{OH}) 2$ and $\mathrm{CuO}$ Nanoparticles. J. Nanopart. Res. 2014, 16 (9), 2584.

(40) Schneider, C. a.; Rasband, W. S.; Eliceiri, K. W. NIH Image to ImageJ: 25 Years of Image Analysis. Nat. Methods 2012, 9 (7), 671675.

(41) Ibupoto, Z. H.; Khun, K.; Lu, J.; Willander, M. The Synthesis of $\mathrm{CuO}$ Nanoleaves, Structural Characterization, and Their Glucose Sensing Application. Appl. Phys. Lett. 2013, 102 (10), 103701103701 .

(42) Bhattacharjee, A.; Ahmaruzzaman, M. Facile Synthesis of 2Dimensional $\mathrm{CuO}$ Nanoleaves and Their Degradation Behavior for Eosin Y. Mater. Lett. 2015, 161, 20-25.

(43) Bhattacharjee, A.; Ahmaruzzaman, M. Green Synthesis of 2D $\mathrm{CuO}$ Nanoleaves (NLs) and Its Application for the Reduction of pNitrophenol. Mater. Lett. 2015, 161, 79-82.

(44) Liang, Z.-H.; Zhu, Y.-J. Microwave-Assisted Synthesis of SingleCrystalline $\mathrm{CuO}$ Nanoleaves. Chem. Lett. 2004, 33 (10), 1314-1315.

(45) Bakshi, M. S. How Surfactants Control Crystal Growth of Nanomaterials. Cryst. Growth Des. 2016, 16 (2), 1104-1133.

(46) Pallipurath, A.; Nicoletti, O.; Skelton, J. M.; Mahajan, S.; Midgley, P. A.; Elliott, S. R. Surfactant-Free Coating of Thiols on Gold Nanoparticles Using Sonochemistry: A Study of Competing Processes. Ultrason. Sonochem. 2014, 21 (5), 1886-1892.

(47) Chen, Q.; Du, G.; Dong, Y.; Cao, Z.; Xie, Z.; Zheng, L. Surfactant Dependent Evolution of Au-Pd Alloy Nanocrystals from Trisoctahedron to Excavated Rhombic Dodecahedron and Multipod: A Matter of Crystal Growth Kinetics. Science Bulletin 2017, 62 (20), $1359-1364$.

(48) Sun, C. Q. Size Dependence of Nanostructures: Impact of Bond Order Deficiency. Prog. Solid State Chem. 2007, 35 (1), 1-159.

(49) Wu, Z.; Yang, S.; Wu, W. Shape Control of Inorganic Nanoparticles from Solution. Nanoscale 2016, 8 (3), 1237-1259.

(50) Xu, H.; Wang, W.; Zhu, W.; Zhou, L.; Ruan, M. HierarchicalOriented Attachment: From One-Dimensional $\mathrm{Cu}(\mathrm{OH}) 2$ Nanowires to Two-Dimensional $\mathrm{CuO}$ Nanoleaves. Cryst. Growth Des. 2007, 7 (12), 2720-2724.

(51) Zhao, Y.; Zhao, J.; Li, Y.; Ma, D.; Hou, S.; Li, L.; Hao, X.; Wang, Z. Room Temperature Synthesis of 2D CuO Nanoleaves in Aqueous Solution. Nanotechnology 2011, 22 (11), 115604-115604.

(52) Xu, X.; Yang, H.; Liu, Y. Self-Assembled Structures of $\mathrm{CuO}$ Primary Crystals Synthesized from $\mathrm{Cu}(\mathrm{CH} 3 \mathrm{COO}) 2-\mathrm{NaOH}$ Aqueous Systems. CrystEngComm 2012, 14 (16), 5289-5289.

(53) Cao, Y.; Liu, S.; Jian, X.; Zhu, G.; Yin, L.; Zhang, L.; Wu, B.; Wei, Y.; Chen, T.; Gao, Y.; Tang, H.; Wang, C.; He, W.; Zhang, W.
Synthesis of High-Purity $\mathrm{CuO}$ Nanoleaves and Analysis of Their Ethanol Gas Sensing Properties. RSC Adv. 2015, 5 (44), 3478834794 .

(54) Zhao, B.; Liu, P.; Zhuang, H.; Jiao, Z.; Fang, T.; Xu, W.; Lu, B.; Jiang, Y. Hierarchical Self-Assembly of Microscale Leaf-Like $\mathrm{CuO}$ on Graphene Sheets for High-Performance Electrochemical Capacitors. J. Mater. Chem. A 2013, 1 (2), 367-373.

(55) Wang, D.; Song, C.; Lv, X.; Wang, Y. Design of Preparation Parameters for Commendable Photocatalytic Properties in $\mathrm{CuO}$ Nanostructures. Appl. Phys. A: Mater. Sci. Process. 2016, 122 (12), 1020.

(56) Mittemeijer, E. J.; Welzel, U. The "State of the Art" of the Diffraction Analysis of Crystallite Size and Lattice Strain. Zeitschrift für Kristallographie 2008, 223 (9), 552-560.

(57) Cullity, B. D. Elements of X-Ray Diffraction, 2nd ed.; AddisonWesley Publishing Company.Inc.: Amsterdam, 1978; p 555.

(58) Andrade, A. B.; Ferreira, N. S.; Valerio, M. E. G. Particle Size Effects on Structural and Optical Properties of BaF2 Nanoparticles. RSC Adv. 2017, 7 (43), 26839-26848.

(59) Borgohain, K.; Singh, J. B.; Rama Rao, M. V.; Shripathi, T.; Mahamuni, S. Quantum Size Effects in CuO Nanoparticles. Phys. Rev. B: Condens. Matter Mater. Phys. 2000, 61 (16), 11093-11096.

(60) Devaraj, M.; Deivasigamani, R. K.; Jeyadevan, S. Enhancement of the Electrochemical Behavior of $\mathrm{CuO}$ Nanoleaves on MWCNTs/ GC Composite Film Modified Electrode for Determination of Norfloxacin. Colloids Surf., B 2013, 102, 554-561.

(61) Anu Prathap, M. U.; Kaur, B.; Srivastava, R. Hydrothermal Synthesis of $\mathrm{CuO}$ Micro-/Nanostructures and Their Applications in the Oxidative Degradation of Methylene Blue and Non-Enzymatic Sensing of Glucose/H2O2. J. Colloid Interface Sci. 2012, 370 (1), 144-154.

(62) Alam, M. M.; Zhao, W.; Zhai, S.; Yusa, S.-i.; Noguchi, H.; Nakashima, K. Fabrication of Copper(II) Oxide Hollow Nanosphere Using $\mathrm{ABC}$ Block Copolymer Templates and Its Application as Anode Materials in Lithium Ion Batteries. Chem. Lett. 2014, 43 (9), $1426-1428$

(63) Talluri, B.; Prasad, E.; Thomas, T. Ultra-Small $(r<2 \mathrm{~nm})$, Stable ( $>1$ year) Copper Oxide Quantum Dots with Wide Band Gap. Superlattices Microstruct. 2018, 113, 600-607.

(64) Mansournia, M.; Arbabi, A. Ammonia Vapor-Assisted Synthesis of $\mathrm{Cu}(\mathrm{OH}) 2$ and $\mathrm{CuO}$ Nanostructures: Anionic $(\mathrm{Cl}-, \mathrm{NO} 3-$, SO4 2-) Influence on the Product Morphology. J. Electron. Mater. 2017, 46 (1), 502-509.

(65) Siddiqui, H.; Qureshi, M. S.; Haque, F. Z. Surfactant Assisted Wet Chemical Synthesis of Copper Oxide $(\mathrm{CuO})$ Nanostructures and Their Spectroscopic Analysis. Optik 2016, 127 (5), 2740-2747.

(66) Born, B.; Krupa, J. D. A.; Geoffroy-Gagnon, S.; Hristovski, I. R.; Collier, C. M.; Holzman, J. F. Ultrafast Charge-Carrier Dynamics of Copper Oxide Nanocrystals. ACS Photonics 2016, 3 (12), 2475-2481.

(67) Velusamy, T.; Liguori, A.; Macias-Montero, M.; Padmanaban, D. B.; Carolan, D.; Gherardi, M.; Colombo, V.; Maguire, P.; Svrcek, V.; Mariotti, D. Ultra-Small CuO Nanoparticles with Tailored EnergyBand Diagram Synthesized by a Hybrid Plasma-Liquid Process. Plasma Processes Polym. 2017, 14 (7), 1600224.

(68) Heinemann, M.; Eifert, B.; Heiliger, C. Band Structure and Phase Stability of the Copper Oxides $\mathrm{Cu} 2 \mathrm{O}, \mathrm{CuO}$, and $\mathrm{Cu} 4 \mathrm{O} 3$. Phys. Rev. B: Condens. Matter Mater. Phys. 2013, 87 (11), 115111.

(69) Gawande, M. B.; Goswami, A.; Felpin, F.-X.; Asefa, T.; Huang, X.; Silva, R.; Zou, X.; Zboril, R.; Varma, R. S. Cu and Cu-Based Nanoparticles: Synthesis and Applications in Catalysis. Chem. Rev. 2016, 116 (6), 3722-3811.

(70) D’Oliveira, J. C.; Al-Sayyed, G.; Pichat, P. Photodegradation of 2- and 3-Chlorophenol in Titanium Dioxide Aqueous Suspensions. Environ. Sci. Technol. 1990, 24 (7), 990-996. 